\title{
Timeliness of Cervical Cancer Diagnosis and Initiation of Treatment in the National Breast and Cervical Cancer Early Detection Program
}

\author{
Vicki B. Benard, Ph.D. ${ }^{1}$, William Howe, B.S. ${ }^{2}$, Janet Royalty, M.S. ${ }^{1}$, William Helsel, M.S. ${ }^{2}$, \\ William Kammerer, B.S. ${ }^{2}$, and Lisa C. Richardson, M.D., M.P.H. ${ }^{1}$
}

${ }^{1}$ Division of Cancer Prevention and Control, Centers for Disease Control and Prevention, Atlanta, Georgia ${ }^{2}$ Information Management Services, Inc., Silver Spring, Maryland

\begin{abstract}
Objectives-To examine time intervals from cervical cancer screening to diagnosis and treatment initiation among low-income and uninsured women in the National Breast and Cervical Cancer Early Detection Program (NBCCEDP) during two consecutive time periods.

Methods-We analyzed NBCCEDP data for women with abnormal Pap tests $(n=100,167)$, from which 1,417 invasive cervical cancers were diagnosed. We examined two time intervals for this study: diagnostic interval (time from abnormal Pap test to the date of definitive diagnosis) and treatment initiation interval (time from definitive diagnosis to treatment initiation) for two time periods: 1996-2002 and 2003-2009. We compared median time intervals for diagnostic and treatment initiation using the Kruskal-Wallis test. Adjusted proportions (predicted marginals) were calculated using logistic regression to examine diagnosis and treatment within program benchmarks ( $\leq 60$ days).
\end{abstract}

Results-Median diagnostic intervals decreased overall by 6 days (54 vs. 48 days, $p<0.001$ ). This decrease in the median diagnostic interval was noted for all variables examined. The median treatment initiation intervals remained stable over the two time periods.

Conclusions-Women screened by the NBCCEDP receive diagnostic follow-up and initiate treatment within preestablished program guidelines.

\section{Introduction}

For cancer screening to be beneficial, it is imperative that patients receive timely and appropriate follow-up for screening-detected abnormalities as a prerequisite to appropriate treatment. Failure to obtain appropriate diagnostic services can have a significant negative effect on health outcomes, as well as costs for both the individual and the healthcare system. ${ }^{1}$ A systematic review of follow-up care after abnormal screening tests for cervical,

\footnotetext{
Address correspondence to: Vicki B. Benard, Ph.D., Epidemiology and Applied Research Branch, Division of Cancer Prevention and Control, Centers for Disease Control and Prevention, 4770 Buford Highway NE, Mailstop K-55, Atlanta, GA 30341, vdb9@ cdc.gov. Disclosure Statement

The authors have no conflicts of interest to report.
} 
breast, and colon cancer showed that $<75 \%$ of women received timely and appropriate follow-up care. ${ }^{2}$ The proportion of women who were followed after abnormal Pap tests varies dramatically across studies, ranging from $7 \%$ to $73 \% .^{2,3}$

Cervical cancer is preventable through early detection and removal of premalignant changes. There are few data to indicate what the optimal diagnostic and treatment intervals are that might ensure the best chances of survival from cervical cancer detected by screening. However, studies have shown that a longer time to treatment, specifically in the medically underserved, results in later stage disease and, thus, poorer survival. ${ }^{4}$ Minority and uninsured women and women from lower socioeconomic backgrounds are less likely to participate in screening, ${ }^{5}$ less likely to have timely and complete follow-up after an abnormal test result, ${ }^{6}$ and more likely to be diagnosed with late-stage disease. ${ }^{7}$

The National Breast and Cervical Cancer Early Detection Program (NBCCEDP) was authorized by Congress in 1990 to reach underserved women. ${ }^{8}$ Since the inception of the program, the NBCCEDP established quality standards to assure timely and complete diagnostic follow-up and treatment initiation for underserved women screened through the program. Legislation for program enhancements that added case management services in 2000 and a Medicaid waiver to support cancer treatment authorized by Congress and implemented in 2003 were expected to improve the ability of programs to meet these standards. ${ }^{9,10}$ Revisions were also made in the reporting system of Pap test results (published in 2002) to more clearly identify cell changes, ${ }^{11}$ along with the introduction of the first evidence-based guidelines for management of abnormal Pap tests (published in 2002). ${ }^{12,13}$ With these improvements both programmatically and reporting, we hypothesized that NBCCEDP quality measures would improve over time (i.e., shortening of time intervals) and that more women would meet the program standards. We used two equal 7year periods, 1996-2002 and 2003-2009, to determine if the intervals have decreased and the percent meeting program standards has improved with implementation of program enhancements and changes within the cytologic management of reporting and guidelines, which would all have been in place by the second time period.

\section{Materials and Methods}

The Centers for Disease Control and Prevention (CDC) implemented cooperative agreements with states, American Indian/Alaska Native tribes, and territories to provide screening, referral, and follow-up services to women through the NBCCEDP, which has been described in detail elsewhere. ${ }^{8}$

Since the program's inception in 1991, the CDC has used a set of standardized data items to monitor screening, diagnostic follow-up, and treatment initiation activities. Women reported demographic characteristics and prior screening history at enrollment. Providers reported dates and results of Pap tests as well as any diagnostic procedures, outcomes, and the date of treatment initiation if applicable. For this study, data from 50 states, the District of Columbia, 13 tribes, and 4 territories were used for the study period 1996-2009. Each woman's county of residence and a U.S. Census data file were used to categorize residence 
at the time of screening as metropolitan, urban, or rural based on the Rural/Urban Continuum Code. ${ }^{14}$ This study was approved by the CDC's Human Subjects Committee.

\section{Study outcomes}

We considered two time intervals for this study: diagnostic interval (time from the first abnormal Pap test to the date of definitive diagnosis, which is specified as the date of the procedure that confirmed a diagnosis) and treatment initiation interval (time from definitive diagnosis to treatment initiation). The diagnostic interval examined the abnormal screening test results defined by the Bethesda reporting system ${ }^{11}$ as low-grade squamous intraepithelial lesion (LSIL), high-grade squamous intraepithelial lesion (HSIL), and squamous cancer ( $\mathrm{SqCa})$. Treatment intervals examined invasive cervical cancers only. Outcomes were examined for two 7-year time periods. Time 1 encompassed 1996-2002, and time 2 encompassed 2003-2009.

\section{Study population}

During the study period from January 1, 1996, through December 31, 2009, 4,402,242 Pap tests were provided to $2,486,028$ women aged $\geq 18$ years (Fig. 1). Among the 2,486,028 women screened, 121,476 women were found to have an abnormal Pap test. A total of 21,309 women were excluded because of missing or invalid service dates or lost to followup or refusal of diagnostic workup or treatment. The final study population consisted of 100,167 women with abnormal screening test results and 1,417 invasive cervical cancers. Time 1 included 33,917 women with abnormal screening test results and 543 cancers, and time 2 included 66,250 women with abnormal screening test results and 874 cancers.

\section{Data analysis}

The distributions of characteristics of the women with abnormal Pap tests, including Pap test result, age, race/ethnicity, and residence, were provided by time period. Chi-square tests with $p$ values were performed to test for association between the characteristics by time period. The diagnostic and treatment interval distributions were highly skewed because of a few outliers. Therefore, we compared medians rather than means to provide a more accurate picture of the true distributions, using the Kruskal-Wallis test to assess statistical significance. We calculated median days and interquartile ranges (IQRs). We created multivariate logistic models stratified by time period, as significant interactions were found between time periods and all covariates included in the final model. We used logistic regression analysis to produce adjusted proportions (predicted marginals, [PM]) to examine diagnosis and treatment within program standards ( $\leq 60$ days), controlling for screening test results, age, race/ethnicity, and residence. In our logistic regression model, timeliness was the dependent variable, and screening test results, age, race/ethnicity, and residence were independent variables. Additionally, the percentages of women meeting the program standards for all years (1996-2009) for diagnosis and treatment were shown and tested for time trends. All statistics were generated using SUDAAN version 9.0 (Research Triangle Institute, Research Triangle Park, NC) and SAS version 9.1 (SAS Institute Inc., Cary, NC). 


\section{Results}

Most women in the study had a less severe abnormal Pap result (LSIL), were < 40 years old, were white, and lived in a metropolitan area (Table 1). Pap tests reported as LSIL increased from $65 \%$ in time 1 to $73 \%$ in time 2 .

Table 2 displays median diagnostic intervals with IQRs and the adjusted proportions for women with an abnormal Pap test being diagnosed within the program standard of 60 days. For the diagnostic interval, the overall median time to diagnosis decreased from 54 to 48 days over the two time periods ( $p<0.001$ ). The biggest improvements in the median diagnostic interval occurred among women who had a Pap test result of $\mathrm{SqCa}$ (33 vs. 22 days, time 1 to time $2, p<0.001$ ), 18-29-year-olds (61 vs. 50 days, time 1 to time $2, p<$ 0.001 ), and black women (61 vs. 50 days, time 1 to time $2 ; p<0.001$ ). With the exception of Asian women, racial and ethnic minorities had longer median times to diagnosis and treatment compared to whites (by 5-10 days in time 1 and 3 days in time 2). Women residing in rural areas had a shorter median diagnostic interval compared to metropolitan and urban areas for both time periods. Women with a diagnosis of cancer had a significantly shorter time than those without cancer for both time periods.

Table 2 also presents adjusted proportions (PM) and 95\% confidence intervals (CI) for women being diagnosed within the program standard of 60 days. Stratified models were constructed to examine the differences within each time period. For the diagnostic interval, all subgroups improved in meeting the 60-day standard. Women with SqCa had the largest improvement in diagnostic interval from time 1 to time 2 of meeting the 60-day program benchmark, from $73 \%$ (95\% CI 69\%-76\%) to 88\% (95\% CI 85\%-90\%), respectively.

Table 3 displays the treatment initiation intervals with IQRs and the adjusted proportions (PM) for women initiating treatment within the program standard of 60 days. For the treatment interval, the overall median time from diagnosis to treatment initiation decreased from 22 to 21 days over the two time periods. The median times improved over time for most subgroups of women; however, there were no statistically significant differences in median days among the variables noted. For the PM, the only statistically significant covariate was Pap test screening result (for time 2).

Figure 2 represents the changes in diagnostic and treatment intervals from 1996 to 2009. Women receiving a diagnosis within 60 days of an abnormal screening test result (program standard) improved from 56\% in 1996 to $71 \%$ in 2009 ( $p<0.001)$. The first significant increase in the percentage meeting standards occurred in 2003 with $60 \%$. The treatment intervals fluctuated across time period.

\section{Discussion}

Overall, 69\% of women with an abnormal Pap test in the NBCCEDP were diagnosed within 60 days after a high-grade diagnosis (68\% HSIL and $83 \%$ SqCa), and $88 \%$ initiated treatment within 60 days after a high-grade diagnosis ( $86 \%$ HSIL and $91 \% \mathrm{SqCa}$ ) (data not shown). The median intervals measured decreased over the two time periods, diagnosis by 6 days and treatment by 1 day. Women with higher-grade lesions were diagnosed in shorter 
time periods, and this improved over time, with a median of only 22 days in time 2 . The NBCCEDP specifies that follow-up systems with appropriate diagnostic and treatment reminders be in place to track and monitor care before implementing screening to ensure that programs meet standards for timeliness and are able to provide feedback to providers. ${ }^{15}$ We found that the proportion of women meeting program standards increased for diagnosis. Most of the improvements occurred in the second time period, when case management and state's Medicaid waivers were fully implemented ${ }^{9,10}$ and improvements in the detection, reporting, and management of cervical cancer were made (published in 2002 and required in NBCCEDP data collection in 2003). ${ }^{11,12}$

Although program standards for timeliness have been met for the majority of women since 1996 (Fig. 2), differences in diagnostic intervals are noted for all subpopulations studied (Table 2). The greatest variations have been observed among racial and ethnic minorities. Median diagnostic intervals for minority women are longer in both time periods, with the exception of Asian women whose intervals are similar to those of white women. However, black and Hispanic women's proportions in meeting the standard have increased more than white women's, so the gap has closed considerably (Hispanic from 7\% to $4 \%$ fewer than white, black from $11 \%$ to $6 \%$ ).

This finding regarding women of racial or ethnic minorities with longer waiting times for diagnosis after cervical cancer screening was also noted in a study of underserved women in California. ${ }^{6}$ Additionally, Richardson et al. ${ }^{16}$ examined the timeliness of breast cancer diagnosis and treatment in the NBCCEDP and also found that minority women had longer median times to diagnosis of breast cancer than did white women. There may be multiple explanations for these findings, including individual characteristics, miscommunication of results, provider characteristics, or the healthcare facilities where minorities receive care. ${ }^{17,18}$ More research is needed within this program to address the racial and ethnic differences observed among a similar underserved population. Although these differences are not clinically significant, women are under psychologic stress waiting for abnormal results to be resolved. ${ }^{19}$

The standards of 60 days for diagnosis and treatment were established at the initiation of the program based on best clinical judgment at the time. To date, there are no scientific data that suggest the 60-day interval for a timely diagnosis is insufficient. Data suggest that the sooner a diagnosis is made and the condition treated, the better the outcome. ${ }^{4}$ Several programs, however, have been challenged to meet the 60-day diagnosis interval performance measure that requires at least $75 \%$ of abnormal screening tests be resolved. Typical challenges encountered by programs include waiting lists for colposcopy appointments, rescheduled appointments, and difficulty referring women. ${ }^{20}$ With the lack of scientific data in the literature and extended conversations with experts in the field, CDC changed the performance measure in November 2009 to 90 days. ${ }^{21}$ Looking at the most recent time period (2003-2009), > 80\% of the programs were meeting the new 90-day requirement.

Over the time period of the study, many advances in the prevention and detection of cervical cancer have occurred. These advances reflect a greater understanding of the natural history of the human papillomavirus (HPV) infection and its role in the development of cervical 
cancer. ${ }^{11,12,22}$ Younger women are more likely to have a high clearance of HPV infections and regression of Pap abnormalities to normal and, therefore, could have a more conservative follow-up approach, as suggested by updated management guidelines. ${ }^{13}$ Our data clearly show that the youngest group (aged 18-29) has a longer time to diagnosis compared to the other age groups and that the median number of days decreases with increasing age (Table 2), suggesting that providers understand this relationship. The median days for treatment for the younger age group are comparable to other ages, however, suggesting that if the lesion is advanced to cancer, they are obtaining timely treatment. ${ }^{23}$

Also in the advances in cervical cancer early detection, the reporting system improved ${ }^{11}$ (published in 2002), and evidence-based management guidelines ${ }^{12}$ (published in 2002) were adopted. The new Bethesda terminology divided the equivocal cytology category to include a high-grade component. Because this category did not exist in the earlier time period, we opted not to include this category in our abnormal results. Additionally, the management guidelines changed, now recommending an immediate colposcopy for an LSIL Pap test result. ${ }^{12}$ Before the published guidelines in 2002, the recommended follow-up for LSIL was a repeat Pap test at 4-6 months for 2 years. ${ }^{24}$ This change in reporting and management guidelines could be why we see an increase in the percent of LSIL Pap results in the second time period. Also of note is the increase in the number of abnormal Pap tests overall from time 1 to time 2 (33,917 and 66,250, respectively) The NBCCEDP began in 1991 with very few states and each year grew in number and funding; this stabilized in 2007. There were a total of 1,117,605 women screened in time 1 and 1,368,423 in time 2 .

In an insured population, investigators found the median length of time from an abnormal Pap test to the recommended colposcopy was 99 days ${ }^{25}$; this was much longer than the 50 days (overall time period) that we observed in the NBCCEDP underserved population without insurance. In an organized screening program in New Zealand, $9 \%$ of women experienced a delay (defined as $>6$ months or 180 days) from high-grade cytology result to diagnosis. ${ }^{26}$ We report a $5 \%$ overall delay over 180 days to diagnosis; this includes lowgrade abnormalities, which generally result in longer times overall (data not shown).

\section{Limitations and strengths}

Our study has several limitations. The program collects only minimal data elements, such as the demographics of the patients; however, we lack information about some patient characteristics that may influence a provider's follow-up and management plan. We are also not able to track the receipt of services outside the program. If a woman received follow-up outside the program, we would not be able to capture this information, and our results could underestimate true follow-up. Additionally, there was a large percentage (17.5\% overall) of women excluded from the cohort because of missing values, lost to follow-up, or refused. Table 1 provides the characteristics of these women; those who were excluded had more LSIL Pap test results, were older ( > 40 years old), and were not white, compared to the cohort studied (for time 1 and time 2). The percentage of women excluded from the study decreased with time (19.4\% time 1 vs. $16.5 \%$ time 2 ). Our results may not be generalizable to the women not served by the program. However, Saraiya et al. ${ }^{27}$ found that in a national 
survey of primary care providers, the NBCCEDP providers were similar to the population in their cervical cancer screening beliefs and practices.

The NBCCEDP is the only national screening program for cervical cancer in the United States. More than 2.6 million women have been screened for cervical cancer since 1991, with a focus on underserved women of racial and ethnic minorities. Although information on behavioral risk factors is lacking, more than 20 years worth of data are available to assess follow-up patterns over time. Moreover, this large collection of clinical data enables a window into the practices of thousands of providers across the United States, operating in a multitude of clinical settings.

\section{Conclusions}

The goal to expand health insurance coverage in the United States has placed new emphasis on the effectiveness of existing public health programs. However, improved access to health services alone does not guarantee effective cancer control and prevention. Even with a national program to help reduce the barriers to early detection, our results still show disparities across racial and ethnic groups. Overall, the program has continued to improve within each subpopulation over time. Results from our study indicate that underserved women screened by the NBCCEDP do receive timely diagnostic follow-up and treatment initiation, as measured by program standards. National standards for all cervical cancer screening programs or similar healthcare delivery systems could be modeled on the program standards to ensure that all women receive adequate follow-up care.

\section{Acknowledgments}

The findings and conclusions in this report are those of the authors and do not necessarily represent the official position of the Centers for Disease Control and Prevention.

\section{References}

1. Bastani R, Yabroff KR, Myers RE, Glenn B. Interventions to improve follow-up of abnormal findings in cancer screening. Cancer. 2004; 101:1188-1200. [PubMed: 15316914]

2. Yabroff KR, Washington KS, Leader A, Neilson E, Mandelblatt J. Is the promise of cancer screening programs being compromised? Quality of follow-up care after abnormal screening results. Med Care Res Rev. 2003; 60:294-331. [PubMed: 12971231]

3. Eggleston KS, Coker AL, Luchok KJ, Meyer TE. Adherence to recommendations for follow-up to abnormal Pap tests. Obstet Gynecol. 2007; 109:1332-1341. [PubMed: 17540805]

4. Ashing-Giwa KT, Tejero JS, Kim J, et al. Cervical cancer survivorship in a population based sample. Gynecol Oncol. 2009; 112:358-364. [PubMed: 19059636]

5. Meissner HI, Yabroff KR, Dodd KW, Leader AE, Ballard-Barbash R, Berrigan D. Are patterns of health behavior associated with cancer screening? Am J Health Promot. 2009; 23:168-175. [PubMed: 19149421]

6. Tabnak F, Muller HG, Wang JL, Zhang W, Howell LP. Timeliness and follow-up patterns of cervical cancer detection in a cohort of medically underserved California women. Cancer Causes Control. 2010; 21:411-420. [PubMed: 20043203]

7. Mandelblatt J, Andrews H, Kerner J, Zauber A, Burnett W. Determinants of late stage diagnosis of breast and cervical cancer: The impact of age, race, social class, and hospital type. Am J Public Health. 1991; 81:646-649. [PubMed: 2014871] 
8. Ryerson, AB.; Benard, VB.; Majors, A. [Accessed on January 2011] National Breast and Cervical Cancer Early Detection Program: Summarizing the first 12 years of partnerships and progress against breast and cervical cancer. Accessed at: http://www.cdc.gov/cancer/nbccedp/pdf/ nationalreport.pdf

9. Lantz PM, Keeton K, Romano L, Degroff A. Case management in public health screening programs: The experience of the National Breast and Cervical Cancer Early Detection Program. J Public Health Manag Pract. 2004; 10:545-555. [PubMed: 15643379]

10. French C, True S, McIntyre R, Sciulli M, Maloy K. State Implementation of the Breast and Cervcial Cancer Prevention and Treatment Act of 2000:A collaborative effort among government agencies. Public Health Rep. 2004; 119:279-285. [PubMed: 15158107]

11. Solomon D, Davey D, Kurman R, et al. The 2001 Bethesda System: Terminology for reporting results of cervical cytology. JAMA. 2002; 287:2114-2119. [PubMed: 11966386]

12. Wright TC Jr, Cox JT, Massad LS, Twiggs LB, Wilkinson EJ. 2001 Consensus guidelines for the management of women with cervical cytological abnormalities. JAMA. 2002; 287:2120-2129. [PubMed: 11966387]

13. Wright TC Jr, Massad LS, Dunton CJ, Spitzer M, Wilkinson EJ, Solomon D. 2006 Consensus guidelines for the management of women with abnormal cervical screening tests. J Low Genit Tract Dis. 2007; 11:201-222. [PubMed: 17917566]

14. U.S. Department of Agriculture. [Accessed on March 2011] Measuring rurality: Rural-Urban Continuum Codes. Accessed at: http://www.ers.usda.gov/Briefing/Rurality/RuralUrbcon

15. Lantz PM, Richardson LC, Sever LE, et al. Mass screening in low-income populations: The challenges of securing diagnostic and treatment services in a national cancer screening program. $\mathrm{J}$ Health Polit Policy Law. 2000; 25:451-471. [PubMed: 10946385]

16. Richardson LC, Royalty J, Howe W, Helsel W, Kammerer W, Benard VB. Timeliness of breast cancer diagnosis and initiation of treatment in the National Breast and Cervical Cancer Early Detection Program, 1996-2005. Am J Public Health. 2010; 100:1769-1776. [PubMed: 20019308]

17. Cardin VA, Grimes RM, Jiang ZD, Pomeroy N, Harrell L, Cano P. Low-income minority women at risk for cervical cancer: A process to improve adherence to follow-up recommendations. Public Health Rep. 2001; 116:608-616. [PubMed: 12196621]

18. Khanna N, Phillips MD. Adherence to care plan in women with abnormal Papanicolaou smears: A review of barriers and interventions. J Am Board Fam Pract. 2001; 14:123-130. [PubMed: 11314919]

19. McCaffery KJ, Irwig L, Turner R, et al. Psychosocial outcomes of three triage methods for the management of borderline abnormal cervical smears: An open randomised trial. BMJ. 2010; 340:b4491. [PubMed: 20179125]

20. NBCCEDP grantees. Communication with NBCCEDP Program.

21. Centers for Disease Control and Prevention. NBCCEDP Monthly Newsletter. 2009.

22. Kulasingam SL, Hughes JP, Kiviat NB, et al. Evaluation of human papillomavirus testing in primary screening for cervical abnormalities: Comparison of sensitivity, specificity, and frequency of referral. JAMA. 2002; 288:1749-1757. [PubMed: 12365959]

23. Yu CK, Chiu C, McCormack M, Olaitan A. Delayed diagnosis of cervical cancer in young women. J Obstet Gynaecol. 2005; 25:367-370. [PubMed: 16091322]

24. Kurman RJ, Henson DE, Herbst AL, Noller KL, Schiffman MH. Interim guidelines for management of abnormal cervical cytology. The 1992 National Cancer Institute Workshop. JAMA. 1994; 271:1866-1869. [PubMed: 8196145]

25. Kuo TM, Benard VB, Berkman ND, Martin CK, Richardson LC. Timing of colposcopy after cervical cytologic abnormalities. Obstet Gynecol. 2010; 115:629-636. [PubMed: 20177296]

26. Priest $P$, Sadler L, Peters J, et al. Pathways to diagnosis of cervical cancer: Screening history, delay in follow up, and smear reading. Br J Obstet Gynaecol. 2007; 114:398-407.

27. Saraiya M, Irwin KL, Carlin L, et al. Cervical cancer screening and management practices among providers in the National Breast and Cervical Cancer Early Detection Program (NBCCEDP). Cancer. 2007; 110:1024-1032. [PubMed: 17628488] 


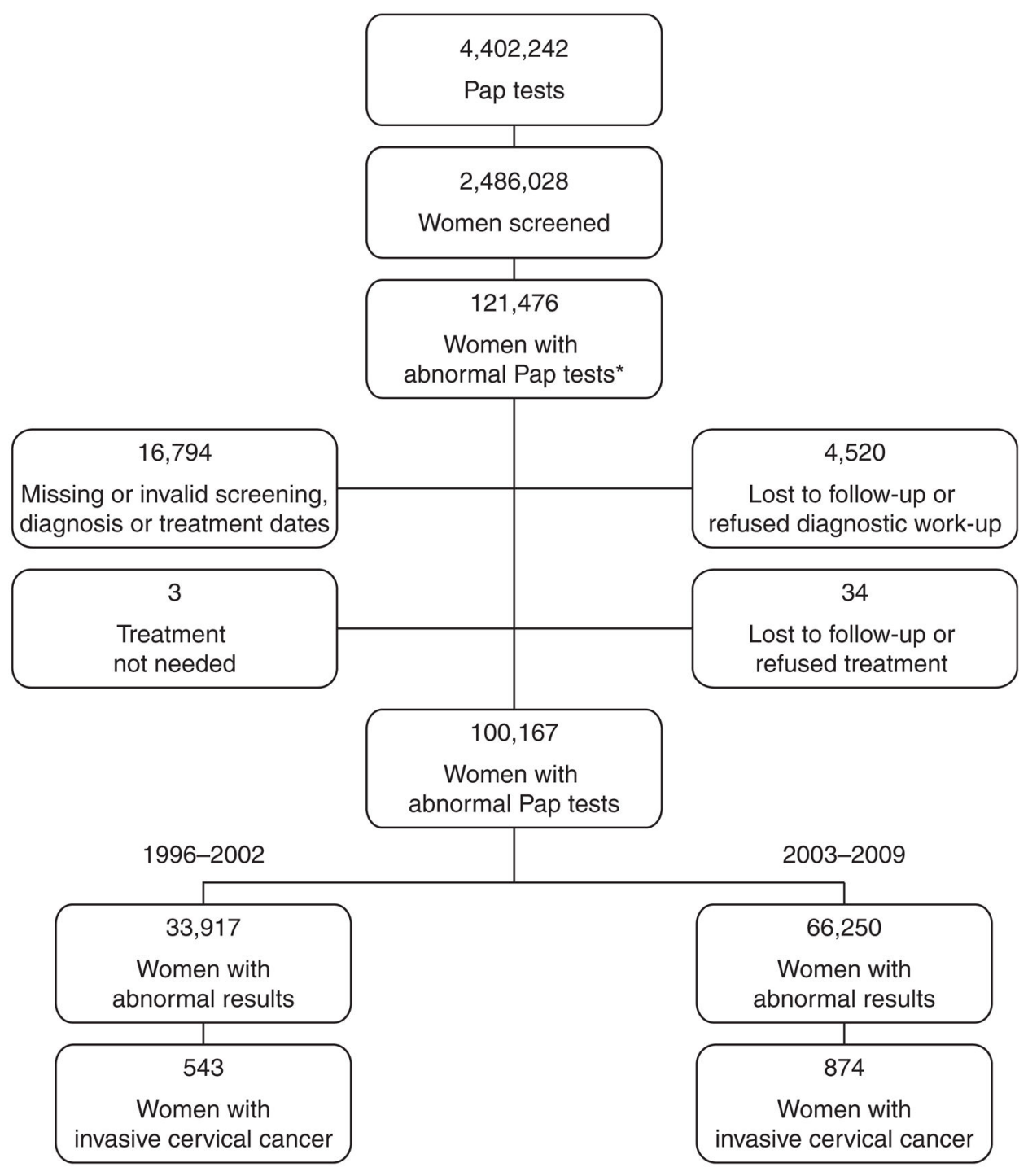

FIG. 1.

Study population in the National Breast and Cervical Cancer Early Detection Program (NBCCEDP), 1996-2009. *Abnormal Pap tests include low-grade squamous intraepithelial lesion, high-grade squamous intraepithelial lesson, and squamous cancer. 


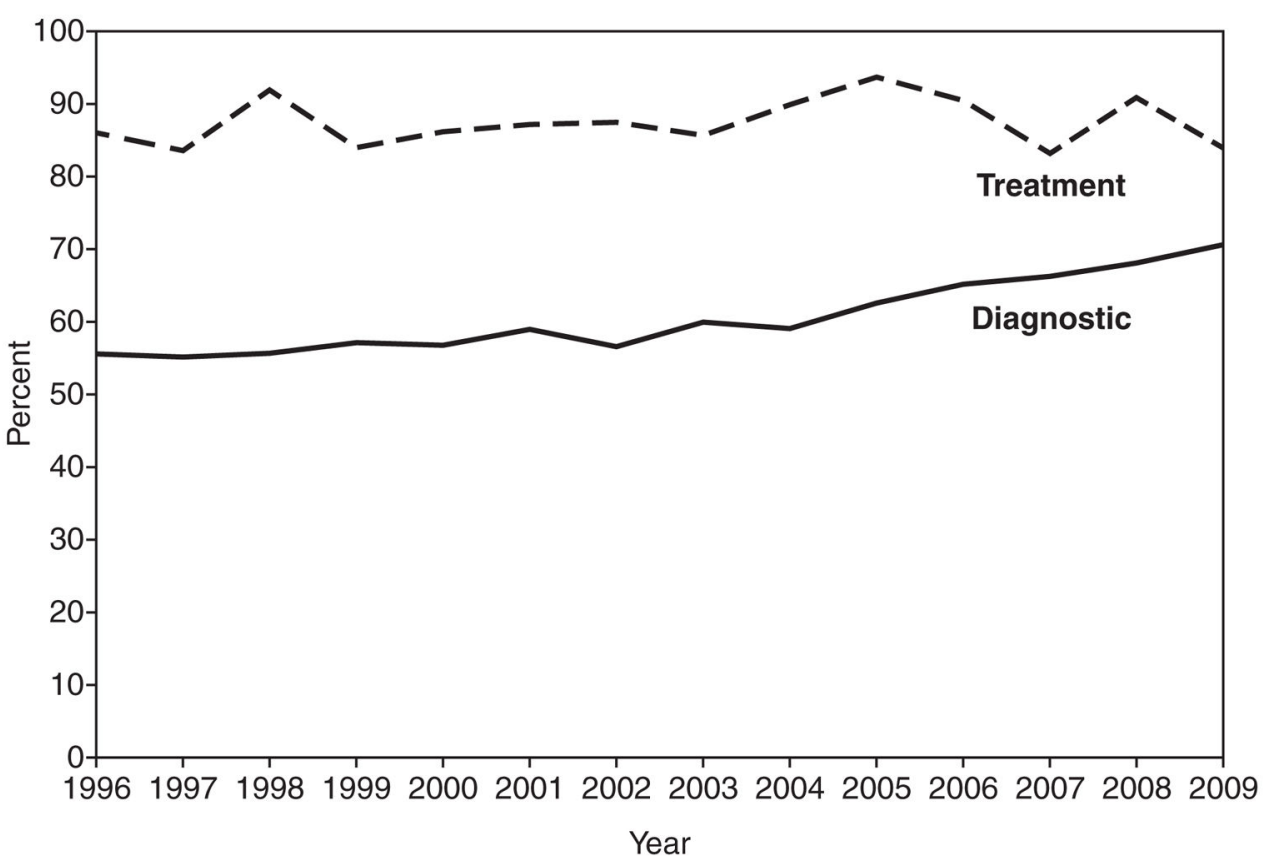

FIG. 2.

Percentage meeting the 60-day diagnostic and treatment intervals in the NBCCEDP, by year 


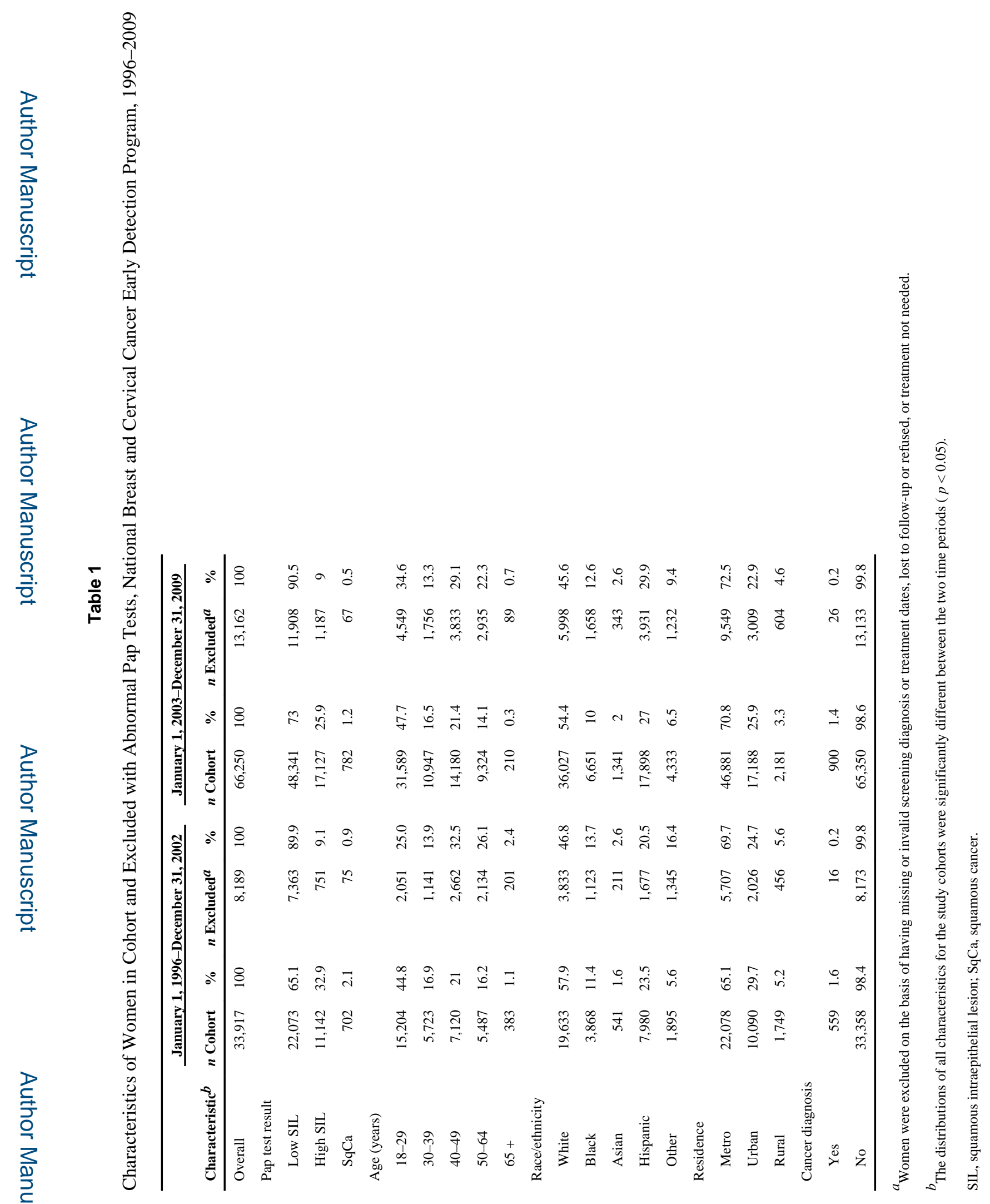

$J$ Womens Health (Larchmt). Author manuscript; available in PMC 2015 August 22. 


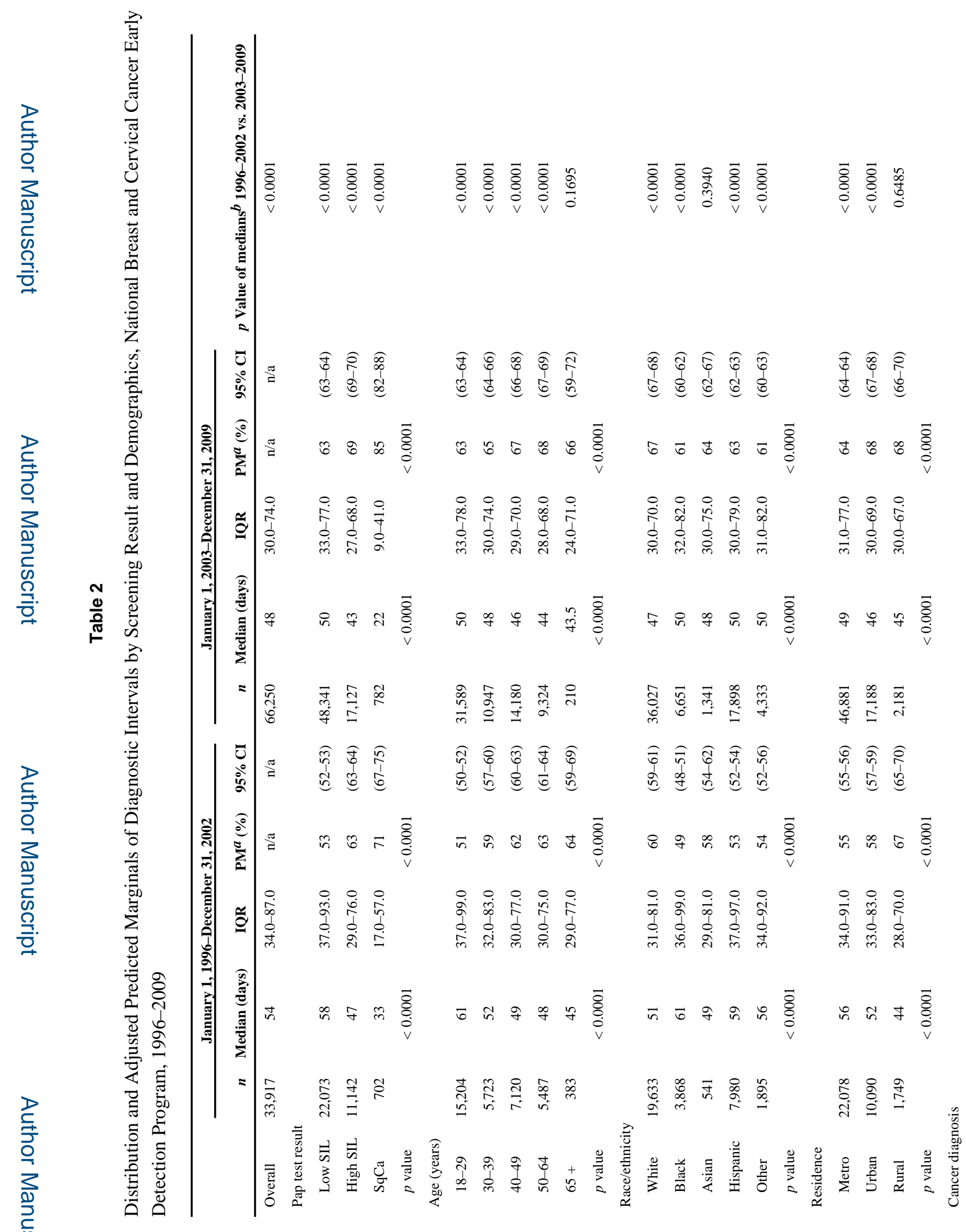




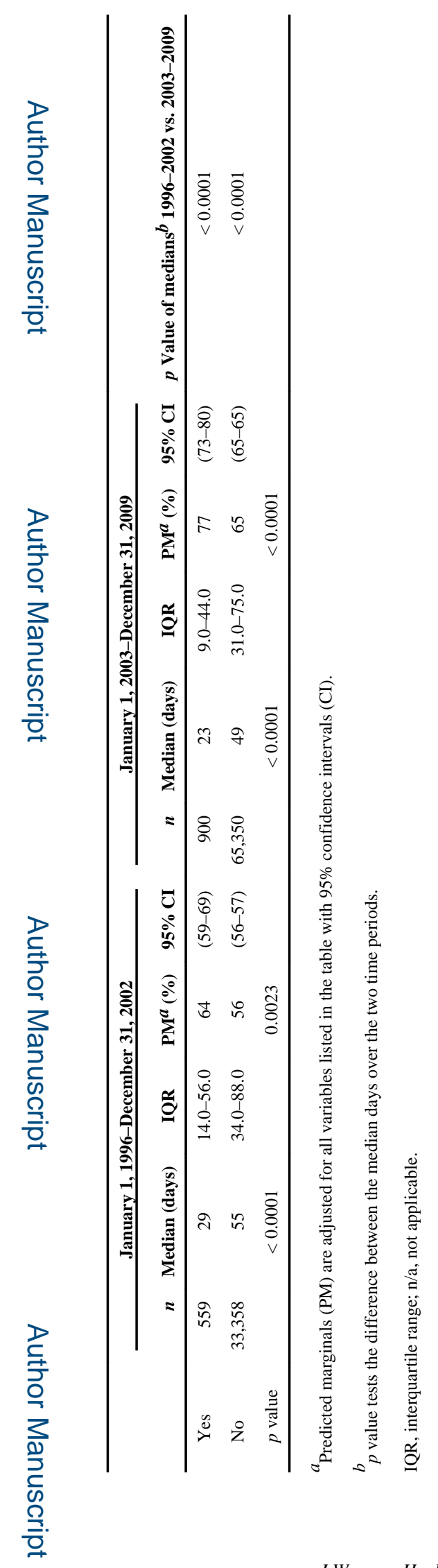

J Womens Health (Larchmt). Author manuscript; available in PMC 2015 August 22. 


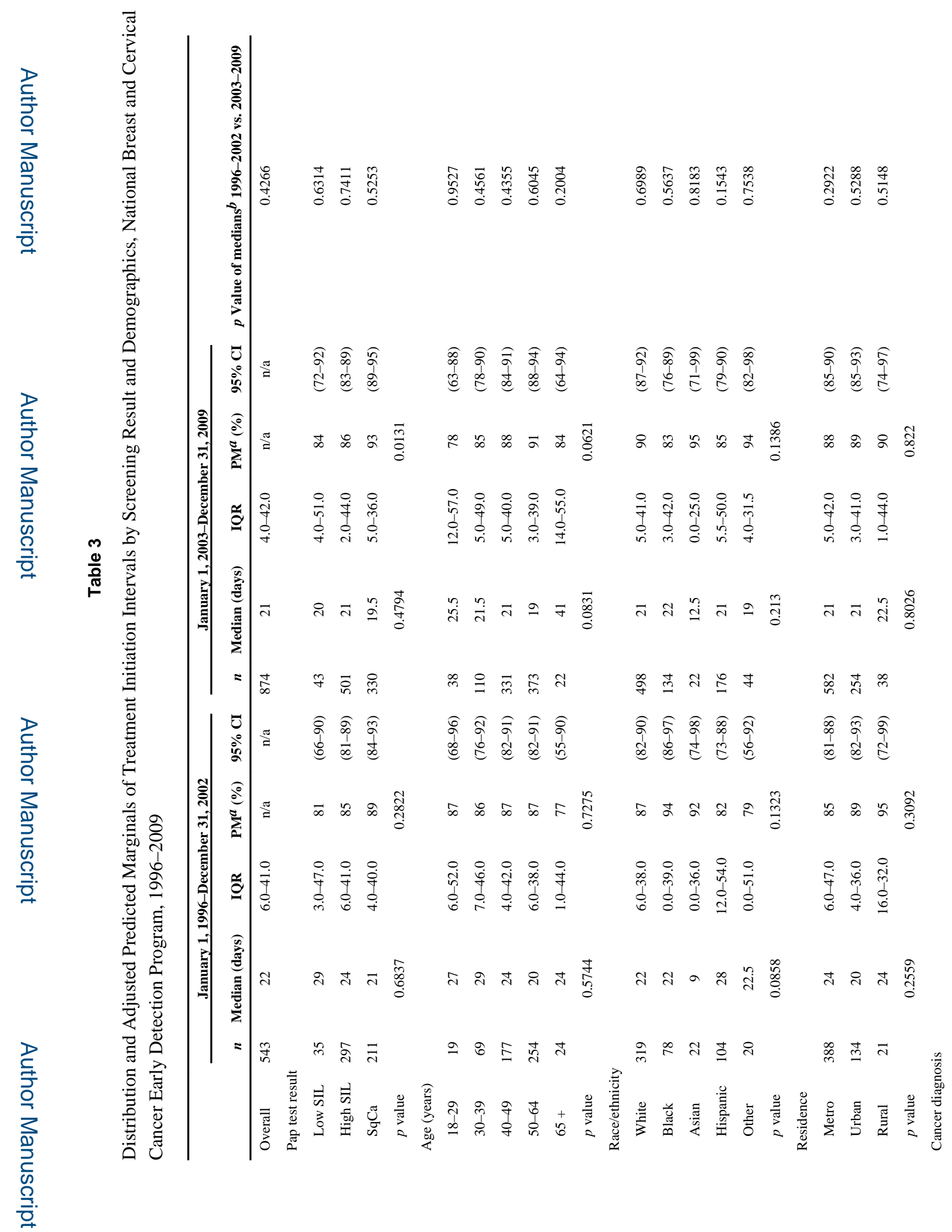

J Womens Health (Larchmt). Author manuscript; available in PMC 2015 August 22. 


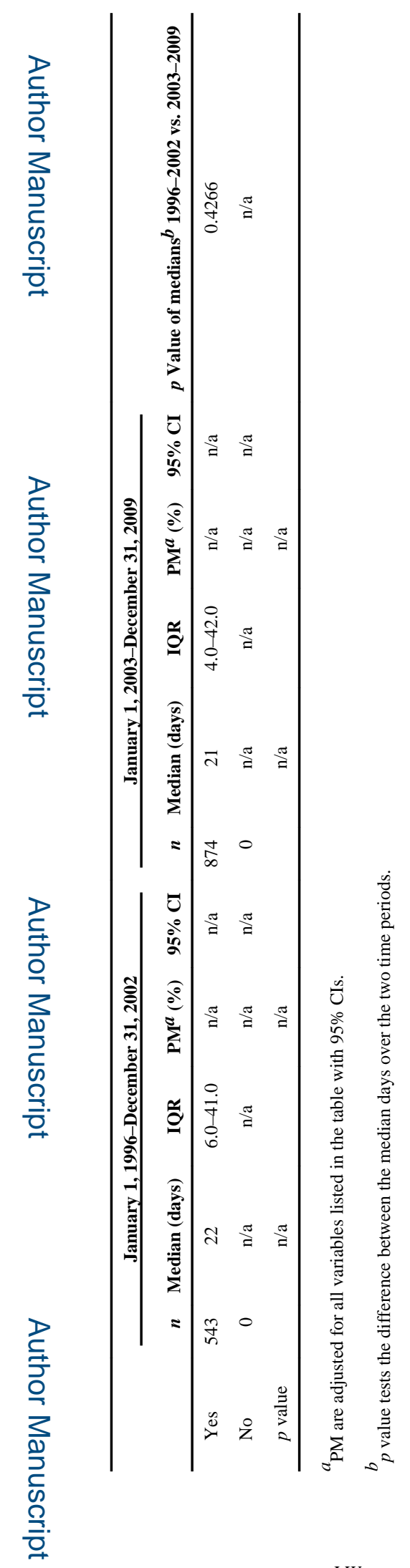

J Womens Health (Larchmt). Author manuscript; available in PMC 2015 August 22. 